\title{
Composite higher derivative theory of gravity
}

\author{
Hans Christian Öttinger (10* \\ ETH Zürich, Department of Materials, HCP F 47.2, CH-8093 Zürich, Switzerland
}

(Received 11 June 2019; accepted 29 January 2020; published 21 February 2020)

\begin{abstract}
We investigate a higher derivative theory that belongs to the class of composite field theories. Starting from the Yang-Mills theory based on the Lorentz group, we express the gauge vector fields in terms of the tetrad decomposition of a space-time metric with a nontrivial coupling constant. The resulting composite gauge theory is a natural candidate for an alternative theory of pure gravity. In the limit of vanishing coupling constant, all classical high-precision tests for theories of gravity are passed. An exact static isotropic solution is found, which is less singular than the Schwarzschild solution of general relativity. Composite field theories come with a natural canonical Hamiltonian formulation and a natural set of constraints, so that they provide an ideal setting for future quantization. Finally, we propose possible couplings of the gravitational field to matter.
\end{abstract}

DOI: 10.1103/PhysRevResearch.2.013190

\section{INTRODUCTION}

The purpose of this work is to elaborate a composite field theory that is a natural candidate for an alternative theory of gravity. Composite theories are obtained by considering the independent variables of some given theory as functions of some more fundamental variables and their derivatives [1,2]. Such composite theories typically involve higher derivatives and are thus prone to instability. The specific structure of composite higher derivative theories, however, comes with natural constraints that eliminate these instabilities.

A particularly interesting composite theory is obtained from the Yang-Mills theory based on the Lorentz group when the gauge vector field is expressed in terms of the tetrad or vierbein variables occurring in the factorization of a spacetime metric. We here explore how the resulting composite theory is related to general relativity and establish it as a promising candidate for an alternative theory of gravity.

The search for alternative theories of gravity is an active field of research because, in spite of its magnificent mathematical elegance and impressive predictive power, Einstein's general theory of relativity has a serious defect: 90 years of unwavering resistance to quantization. Moreover, the ongoing search for "dark energy" might point to a lack of our understanding of gravity on the largest scales (see [3], also for a broad review of extended theories of gravity).

\section{COMPOSITE THEORIES}

The basic idea of composite theories has been developed in the context of mechanical systems [1,2]. However, the finitedimensional systems have been taken so general that spatially discretized versions of the field theories we are interested in

\footnotetext{
*hco@mat.ethz.ch; www.polyphys.mat.ethz.ch

Published by the American Physical Society under the terms of the Creative Commons Attribution 4.0 International license. Further distribution of this work must maintain attribution to the author(s) and the published article's title, journal citation, and DOI.
}

are included. In a brief review of composite theories, we here restrict ourselves to features that carry over directly to field theories.

The development of composite theories is most conveniently based on a Lagrangian $L(q, \dot{q})$, which implies the evolution equations for the variables $q$ and the corresponding Hamiltonian formulation by standard procedures (variational principle, Legendre transformation). We say that the Lagrangian $L(q, \dot{q})$, or the corresponding Hamiltonian $H(q, p)$, serves as our "workhorse theory." We further assume that the variables $q$ can be expressed as $q(\bar{q}, \dot{\bar{q}})$ in terms of fewer, more basic variables $\bar{q}$ and their time derivatives. We refer to the relation $q(\bar{q}, \dot{\bar{q}})$ as the "composition rule" and typically assume a linear dependence of $q$ on $\dot{\bar{q}}$. Composite theories offer an epistemologically appealing setup because they may be interpreted as gain in understanding by recognizing more fundamental variables behind successful theories. A composite theory is simply defined by the Lagrangian $\bar{L}(\bar{q}, \dot{\bar{q}}, \ddot{\bar{q}})$ that is obtained by expressing the original Lagrangian in terms of the new variables.

A composite theory with Lagrangian $\bar{L}(\bar{q}, \dot{\bar{q}}, \ddot{\bar{q}})$ typically leads to fourth-order evolution equations. A general Hamiltonian framework for such higher derivative theories has been proposed in a classical paper by Ostrogradsky [4] and applied to the special case of composite theories in [1] (for more background information on general higher derivative theories see [5-7], for some applications see [8-13] and references therein). In this classical approach, $\bar{q}$ and $\dot{\bar{q}}$ serve as configurational variables and the corresponding conjugate momenta involve second and third time derivatives of $\bar{q}$. A more elegant Hamiltonian formulation of composite theories can be based on $q$ and $\bar{q}$ as configurational variables and the corresponding conjugate momenta $p$ (from the workhorse theory) and $\bar{p}$ (containing third derivatives of $\bar{q}$ ) [2]. The resulting Hamiltonian is linear in the momentum $\bar{p}$. Therefore, this Hamiltonian cannot be bounded from below and we expect the occurrence of instabilities.

The space for the elegant Hamiltonian formulation of the composite theory $(q, \bar{q}, p, \bar{p})$ is much larger than the space 
for the workhorse theory $(q, p)$, which is actually combined with the canonical space of the more fundamental variables. This observation seems to be at variance with the idea that the solutions of the workhorse theory should be restricted by prescribing trial functions of the form $q(\bar{q}, \dot{\bar{q}})$ in terms of fewer variables. Both this seeming discrepancy and the problem of instabilities are resolved by constraints, which play a key role in higher derivative theories.

The composition rule $q(\bar{q}, \dot{\bar{q}})$ does not only provide the canonical variables $\bar{q}$ for the Hamiltonian formulation, but also implies a natural set of primary constraints expressing its consistency. By requiring dynamic consistency of the constraints, the primary constraints lead to secondary and successively higher constraints. The examples of [2] show that (i) the hierarchy of constraints ends with the constraints $\bar{p}=0$, and (ii) the entire set of constraints is so large that the composite theory has fewer degrees of freedom than the workhorse theory. The constraints $\bar{p}=0$ make the Hamiltonian bounded from below and therefore eliminate all concerns about instabilities. They furthermore imply that we can solve the workhorse theory for $q(t)$ in a first step and then, in a postprocessing step, find the solutions that can be represented in the form $q(t)=q(\bar{q}(t), \dot{\bar{q}}(t))$. This procedure is most transparent for steady states, where we are interested in the time-independent solutions of the workhorse theory that can be written in the form $q(\bar{q}, 0)$.

The elegant Hamiltonian version of composite higher derivative theories and their usual Ostrogradsky formulation are not equivalent. In particular, the dimensions of the underlying spaces, the structure of the constraints, and the arguments for obtaining the primary constraints are different. Nevertheless, they both lead to the above postprocessing strategy for restricting the solutions of the workhorse theory and for eliminating instabilities from the composite higher derivative theory.

Composite theories and gauge theories share some common features. Both kinds of theories become simpler and more elegant when considered in an unphysically large space (for the composite theories, one gains a natural Hamiltonian structure). For both types of theories, the physically relevant states are selected by imposing constraints.

\section{YANG-MILLS THEORY BASED ON THE LORENTZ GROUP}

Yang-Mills theories play a central role in fundamental particle physics. The presently best theories of weak and strong interactions are Yang-Mills theories based on the special unitary gauge groups $\mathrm{SU}(2)$ and $\mathrm{SU}(3)$, respectively. Electromagnetism may be considered as the Yang-Mills theory associated with the commutative group U(1). In this section, we provide the relevant details on the Yang-Mills theory based on the Lorentz group $\mathrm{SO}(1,3)$ which, in the subsequent sections, is explored as the workhorse theory for a composite higher derivative theory of gravity.

\section{A. Basic notation}

For developing Yang-Mills theories, including our workhorse for gravity, we consider a fixed background
TABLE I. Correspondence between label $a$ for the base vectors of the six-dimensional Lie algebra $\operatorname{so}(1,3)$ and ordered pairs $(\tilde{\kappa}, \tilde{\lambda})$ of space-time indices.

\begin{tabular}{lcccccc}
\hline \hline$a$ & 1 & 2 & 3 & 4 & 5 & 6 \\
\hline$(\tilde{\kappa}, \tilde{\lambda})$ & $(0,1)$ & $(0,2)$ & $(0,3)$ & $(2,3)$ & $(3,1)$ & $(1,2)$ \\
\hline \hline
\end{tabular}

Minkowski space where $x^{0}=c t$ is the product of the speed of light and time, $x^{1}, x^{2}, x^{3}$ are the spatial coordinates, and $\eta_{\mu \nu}=\eta^{\mu \nu}$ denotes the Minkowski metric [with signature $(-,+,+,+)]$. The Minkowski metric, which is its own inverse, is always used for raising or lowering space-time indices throughout this paper.

\section{B. Lorentz group}

As the subsequent development strongly relies on the Lorentz group $\mathrm{SO}(1,3)$, we briefly summarize its most important properties. The Lorentz group consists of the real $4 \times 4$ matrices that leave the Minkowski metric invariant. Our focus is on its Lie algebra $\operatorname{so}(1,3)$, which actually characterizes the restricted Lorentz group (the connected component containing the identity element). This Lie algebra is six dimensional; we here choose six natural base vectors, three of which generate the Lorentz boosts in the coordinate directions and the other three generate rotations around the coordinate axes. The presence of boosts makes the Lorentz group noncompact. It is convenient to switch back and forth between the labels $a=1, \ldots 6$ for all six generators and the pairs $(0,1),(0,2)$, $(0,3)$ for the boosts and $(2,3),(3,1),(1,2)$ for the rotations according to Table I. With these index conventions, we can introduce the generators of the restricted Lorentz group, or a set of convenient base vectors for its Lie algebra, as

$$
T_{\lambda}^{a \kappa}=\delta_{\lambda}^{\tilde{\kappa}} \eta^{\tilde{\lambda} \kappa}-\eta^{\tilde{\kappa} \kappa} \delta_{\lambda}^{\tilde{\lambda}},
$$

so that $T^{a}{ }_{\kappa \lambda}=\eta_{\kappa \kappa^{\prime}} T^{a \kappa^{\prime}}{ }_{\lambda}$ and $T^{a \kappa \lambda}=\eta^{\lambda \lambda^{\prime}} T^{a \kappa}{ }_{\lambda^{\prime}}$ are both antisymmetric in $\kappa$ and $\lambda$ (and also in $\tilde{\kappa}$ and $\tilde{\lambda}$, if we do not restrict ourselves to the ordered index pairs listed in Table I).

Contrary to the standard Yang-Mills theories based on the special unitary gauge groups, we here need to distinguish between upper and lower Lie algebra labels. The identification of the labels for the base vectors of the Lie algebra with pairs of space-time indices in Table I suggests to use the matrix

$$
K^{a b}=\eta^{\tilde{\kappa}_{a} \tilde{\kappa}_{b}} \eta^{\tilde{\lambda}_{a} \tilde{\lambda}_{b}},
$$

for raising the labels of the Lie algebra. Note that $K^{a b}$ is the diagonal $6 \times 6$ matrix with diagonal elements $(-1,-1,-1,1,1,1)$, which is its own inverse, denoted by $K_{a b}$. The matrix $K_{a b}$ is used for lowering Lie algebra labels.

With the definition (1), we can identify the structure constants $f_{c}^{a b}$ characterizing the commutators

$$
\left[T^{a}, T^{b}\right]=f_{c}^{a b} T^{c} .
$$

Moreover, the matrix $K^{a b}$ is recognized to be a multiple of the Cartan-Killing metric

$$
f_{d}^{a c} f_{c}^{b d}=-4 K^{a b}
$$


The quantities $K^{a b}$ and $f_{c}^{a b}$ are related to the properties of the traces of the generators (1) according to

$$
\begin{gathered}
\operatorname{tr}\left(T^{a}\right)=0, \quad-\operatorname{tr}\left(T^{a} T^{b}\right)=2 K^{a b}, \\
-\operatorname{tr}\left(T^{a} T^{b} T^{c}\right)=-\frac{1}{2} \operatorname{tr}\left(\left[T^{a}, T^{b}\right] T^{c}\right)=f_{d}^{a b} K^{d c}=f^{a b c} .
\end{gathered}
$$

The structure constants can be specified as follows: $f^{a b c}$ is $1(-1)$ if $(a, b, c)$ is an even (odd) permutation of $(4,5,6)$, $(1,3,5),(1,6,2)$, or $(2,4,3)$ and vanishes otherwise. These structure constants satisfy the Jacobi identity

$$
f_{a}^{s b} f_{s}^{c d}+f_{a}^{s c} f_{s}^{d b}+f_{a}^{s d} f_{s}^{b c}=0 .
$$

As the Cartan-Killing metric (4) is nondegenerate, the Lie algebra of the Lorentz group is a direct sum of simple Lie algebras. This observation has important consequences for the general theory of representations for the Lorentz group [14].

\section{Action and field equations}

We can now build a standard Yang-Mills theory based on the restricted Lorentz group (see, e.g., Sec. 15.2 of [15], Chap. 15 of [16], or [17]). The basic variables of a Yang-Mills theory are the four-vector potentials $A_{a v}$, which characterize a fourvector field $A_{a v} T^{a}$ in the Lie algebra. If we further introduce the field tensor

$$
F_{a \mu \nu}=\frac{\partial A_{a v}}{\partial x^{\mu}}-\frac{\partial A_{a \mu}}{\partial x^{\nu}}+\tilde{g} f_{a}^{b c} A_{b \mu} A_{c v},
$$

where the parameter $\tilde{g}$ (or coupling constant) characterizes the strength of the interactions, Yang-Mills theory is defined by the action

$$
I=\int\left(-\frac{1}{4} F_{a \mu \nu} F^{a \mu \nu}\right) d^{4} x .
$$

From the stationarity of this action under variations of $A_{a v}$, we obtain the following field equations:

$$
\frac{\partial F_{a}^{\mu \nu}}{\partial x^{\mu}}+\tilde{g} f_{a}^{b c} A_{b \mu} F_{c}^{\mu \nu}=0 .
$$

The discussion of gauge transformations is postponed to the next section, so that we can benefit from a broader perspective. In particular, we wish to identify the origin of gauge invariance in the setting of composite theories.

\section{FROM METRIC TENSOR TO GAUGE VECTORS}

The composite theory that we want to study employs the Yang-Mills theory based on the Lorentz group as a workhorse theory. We now wish to formulate and motivate a natural composition rule for the corresponding four-vector variables $A_{a v}$ and to discuss the roots of their gauge transformation behavior.

\section{A. Composition rule for four-vector potentials}

We propose the following definition of the four-vector variables $A_{a v}$ in terms of more basic variables,

$$
\begin{aligned}
A_{a \nu} T_{\kappa \lambda}^{a}= & \frac{1}{2} \bar{b}_{\kappa}^{\mu}\left(\frac{\partial g_{\mu \nu}}{\partial x^{\mu^{\prime}}}-\frac{\partial g_{\mu^{\prime} v}}{\partial x^{\mu}}\right) \bar{b}_{\lambda}^{\mu^{\prime}} \\
& +\frac{1}{2 \tilde{g}} \frac{\partial b^{\kappa^{\prime}}}{\partial x^{\nu}}\left(\bar{b}_{\kappa}^{\mu} \eta_{\kappa^{\prime} \lambda}-\bar{b}_{\lambda}^{\mu} \eta_{\kappa^{\prime} \kappa}\right),
\end{aligned}
$$

where $\tilde{g}$ is a dimensionless coupling constant and the righthand side is antisymmetric in $\kappa$ and $\lambda$, so that it can be represented in terms of the generators of the Lorentz group.

The various tensor variables occurring in Eq. (11) can be explained by considering the following decomposition of a symmetric matrix,

$$
g_{\mu \nu}=\eta_{\kappa \lambda} b_{\mu}^{\kappa} b_{\nu}^{\lambda},
$$

where the matrix $g_{\mu \nu}$ possesses all the properties of a spacetime metric of general relativity. This decomposition may be interpreted as a general transformation of the Minkowski metric of a freely falling system to the background space, where an arbitrariness of the decomposition arises from the (gauge) freedom of performing Lorentz transformations that do not affect the Minkowski metric.

The nondegenerate $4 \times 4$ matrix $b^{\kappa}{ }_{\mu}$ consists of four fourvector fields labeled by $\kappa$ ( $\mu$ is the four-vector index). These vector fields, which are known as tetrad or vierbein variables in general relativity, serve as our fundamental variables for a composite field theory of gravity (see, for example, Sec. 12.5 of [18]). The metric $g_{\mu \nu}$ is given in terms of $b^{\kappa}{ }_{\mu}$ by Eq. (12), and $\bar{b}=b^{-1}$ is the inverse of $b^{\kappa}{ }_{\mu}$. The inverse $\bar{b}$ allows us to decompose also the inverse metric tensor as

$$
\bar{g}^{\mu \nu}=\eta^{\kappa \lambda} \bar{b}_{\kappa}^{\mu} \bar{b}_{\lambda}^{\nu} .
$$

We use the symbols $\bar{b}$ and $\bar{g}$ because these matrices are not obtained by lowering and raising the indices of $b$ and $g$ by the Minkowski metric, but by forming inverses. It is verified below that, for any nonzero value of the coupling constant $\tilde{g}$, the variables $A_{a v}$ indeed acquire the desired character of gauge vector fields from the ambiguity of the decomposition (12).

For any $a, A_{a v}$ represents a four-vector under Lorentz transformations. A direct calculation based on Eq. (11) shows that vector behavior under general space transformations is obtained only for $\tilde{g}=1$. As we are going to assume small values of the coupling constant $(|\tilde{g}| \ll 1)$, the composite theory of gravity proposed in this paper deviates from general relativity.

We here use the tensor $g_{\mu \nu}$ to pass from velocities to momenta. Such a tensorial relationship may be interpreted as a relativistic anisotropy of mass. This is a weaker requirement than the usual postulate of a metric in general relativity. In particular, $g_{\mu \nu}$ is assumed to be a tensor under Lorentz transformations only, not under general coordinate transformations.

As all the quantities appearing in Eqs. (12) and (13) are dimensionless, $A_{a v}$ must have the dimension of inverse length. Note that the field tensor has dimensions of length ${ }^{-2}$, so that the standard Yang-Mills action (9) turns out to be dimensionless. The general coupling constant in Eq. (11) sacrifices the general vector behavior of $A_{a v}$, but preserves (and fully explores) the gauge transformation behavior of the Lorentz vector $A_{a v}$.

\section{B. Motivation and discussion of composition rule}

In the following, we wish to motivate the transformation rule (11) from tetrad variables to gauge-vector fields, which is a cornerstone of our development of a composite higher derivative theory of gravity. We begin this motivation by 
considering the relevant connections between the tetrads at different points $x^{\mu}$. The definition of the relevant connection variables is analogous to that of the Ashtekar variables proposed for a canonical approach to gravity in the context of dreibein variables [19,20]. We postulate the following condition for matching tetrads at different space-time points:

$$
\frac{\partial b^{\kappa}{ }_{\mu}}{\partial x^{\nu}}-X_{\mu \nu}^{\mu^{\prime}} b_{\mu^{\prime}}+Y_{\nu \kappa^{\prime}}^{\kappa} b_{\mu}^{\kappa^{\prime}}=0,
$$

where the Levi-Civita connection $X_{\mu \nu}^{\mu^{\prime}}$ and the Levi-Civita spin connection $Y_{v \kappa^{\prime}}^{\kappa}$ remain to be determined (for simplicity, we restrict ourselves to the torsion-free case). In order to do so, we consider the corresponding changes of the metric

$$
\frac{\partial g_{\mu \mu^{\prime}}}{\partial x^{\nu}}-X_{\mu \nu}^{\rho} g_{\rho \mu^{\prime}}-X_{\mu^{\prime} \nu}^{\rho} g_{\mu \rho}+\left(Y_{\kappa \nu \lambda}+Y_{\lambda \nu \kappa}\right) b^{\kappa}{ }_{\mu} b_{\mu^{\prime}}^{\lambda}=0,
$$

where we have used our standard convention for lowering space-time indices, $Y_{\kappa \nu \lambda}=\eta_{\kappa \kappa^{\prime}} Y_{\nu \lambda}^{\kappa^{\prime}}$. The Levi-Civita connection $X_{\mu \nu}^{\mu^{\prime}}$ associated with the metric $g_{\mu \nu}$ is given by the Christoffel symbols

$$
X_{\mu \nu}^{\mu^{\prime}}=\Gamma_{\mu \nu}^{\mu^{\prime}}=\frac{1}{2} \bar{g}^{\mu^{\prime} \rho}\left(\frac{\partial g_{\varrho \mu}}{\partial x^{\nu}}+\frac{\partial g_{\rho \nu}}{\partial x^{\mu}}-\frac{\partial g_{\mu \nu}}{\partial x^{\rho}}\right),
$$

and $Y_{\kappa \nu \lambda}$ must be antisymmetric in $\kappa$ and $\lambda$,

$$
Y_{\kappa \nu \lambda}=-Y_{\lambda \nu \kappa}
$$

Equation (14) determines $Y_{v \lambda}^{\kappa}$ in terms of tetrad variables and Christoffel symbols:

$$
Y_{\nu \lambda}^{\kappa}=\left(\Gamma_{\mu \nu}^{\mu^{\prime}} b_{\mu^{\prime}}^{\kappa}-\frac{\partial b_{\mu}^{\kappa}}{\partial x^{\nu}}\right) \bar{b}_{\lambda}^{\mu} .
$$

If we write $Y_{\kappa \nu \lambda}$ in terms of tetrad variables and metric,

$$
\begin{aligned}
Y_{\kappa \nu \lambda}= & \frac{1}{2} \bar{b}_{\kappa}^{\mu}\left(\frac{\partial g_{\mu \nu}}{\partial x^{\mu^{\prime}}}-\frac{\partial g_{\mu^{\prime} \nu}}{\partial x^{\mu}}\right) \bar{b}_{\lambda}^{\mu^{\prime}} \\
& +\frac{1}{2} \frac{\partial b^{\kappa^{\prime}} \mu}{\partial x^{\nu}}\left(\bar{b}_{\kappa}^{\mu} \eta_{\kappa^{\prime} \lambda}-\bar{b}_{\lambda}^{\mu} \eta_{\kappa^{\prime} \kappa}\right),
\end{aligned}
$$

the required antisymmetry (17) of $Y_{\kappa \nu \lambda}$ in $\kappa$ and $\lambda$ becomes obvious. This antisymmetry implies that we can interpret $Y_{\kappa \nu \lambda}$ as a vector field in the Lie algebra of the Lorentz group so that we can represent it in terms of the six base vectors defined in Eq. (1). We thus arrive at the desired motivation of our composition rule (11).

Our heuristic motivation would suggest the particular choice $\tilde{g}=1$, but we here simply take Eq. (11) as a more general composition rule. As, in our development of a composite field theory of gravity, Eq. (11) has the logical status of the definition of a composition rule, we are free to introduce the additional parameter $\tilde{g}$. The presence of $\tilde{g}$ makes the full theory significantly different from previous proposals of Yang-Mills gravity based on the Lorentz group and turns out to be crucial for reproducing the predictions of general relativity that have been tested with high precision.

The curvature tensor, which plays an important role in general relativity, can be expressed in terms of first and second derivatives of the metric $g_{\mu \nu}$ and hence alternatively of the tetrad variables $b_{\mu}^{\kappa}$. It is remarkable that, for $\tilde{g}=1$, the
Ricci curvature tensor can be expressed in a simple algebraic form in terms of the tetrad variables $b^{\kappa}{ }_{\mu}$ and the field tensor $F_{a \mu \nu}$ associated with the vector potentials $A_{a v}$ introduced in Eq. (11):

$$
R_{\nu}^{\mu}=-\bar{b}_{\kappa}^{\mu} \bar{b}_{\lambda}^{v^{\prime}} \eta^{\kappa \tilde{\kappa}} \eta^{\lambda \tilde{\lambda}} F_{\tilde{\kappa} \tilde{\lambda}, v^{\prime} v}=\Delta^{a \mu v^{\prime}} F_{a v^{\prime} \nu},
$$

where we have introduced the tensors

$$
\Delta^{a \mu \nu}=T^{a \kappa \lambda} \bar{b}_{\kappa}^{\mu} \bar{b}_{\lambda}^{\nu},
$$

which can be interpreted as the base vectors of the Lie algebra transformed from a freely falling to the background space. Equation (20) includes either a summation over the six values of the Lie algebra label $a$ or over all 16 combinations of the space-time indices $\tilde{\kappa}$ and $\tilde{\lambda}$, where we define the following antisymmetric generalization to arbitrary index pairs $F_{\tilde{\lambda} \tilde{\kappa}, \mu \nu}=$ $-F_{\tilde{\kappa} \tilde{\lambda}, \mu \nu}$. The gauge-invariant tensor $R^{\mu}{ }_{v}$ is expected to play an important role for the coupling of the gravitational field to matter [see Eqs. (51) and (52) in the Conclusions].

Of course, connections between Yang-Mills theory and gravity have been explored before. Only two years after the proposal of Yang-Mills theory [21], Utiyama established a connection between the gravitational field and the YangMills theory based on the Lorentz group [22]. That paper is at the origin of what is now known as gauge gravitation theory $[3,23]$. The original proposal by Utiyama has been criticized as "unnatural" by Yang (see footnote 5 of [24]) and Yang's allegedly more natural proposal [24] has itself been massively criticized in Chap. 19 of [25]. The key innovation of this paper is the more general transformation (11) between the metric of general relativity and the vector potential of Yang-Mills theory.

To summarize our developments in the language of chess, the introduction of the various variables in this section is a standard opening. The elements from the popular Sicilian Defense (or, Einstein's general relativity) are mentioned only very briefly, whereas the moves from the more aggressive Latvian Gambit (or, Einstein-Cartan theory) are described more explicitly. There is only one bold move that does not occur in the standard opening theory: the introduction of the factor $1 / \tilde{g}$ in Eq. (11), which is allowed to deviate from unity. This move is crucial for the further development of a successful composite higher derivative theory of gravity which, within the scope of this paper, can only be performed on the level of a middle game. The more challenging end game must be left to future work.

\section{Gauge transformations}

Let us consider the gauge freedom associated with the decomposition (12) in more detail. As Lorentz transformations leave the Minkowski metric in Eq. (12) invariant, we can easily identify the infinitesimal gauge transformations of $b$ and $\bar{b}$ that leave the metric $g_{\mu \nu}$ invariant,

$$
\delta b^{\kappa}{ }_{\mu}=-\tilde{g} \Lambda_{a} T^{a \kappa}{ }_{\lambda} b_{\mu}^{\lambda}, \quad \delta \bar{b}_{\kappa}^{\mu}=\tilde{g} \Lambda_{a} T^{a \lambda}{ }_{\kappa} \bar{b}_{\lambda}^{\mu},
$$

where $\tilde{g} \Lambda_{a}$ characterizes infinitesimal Lorentz transformations. By means of the definition (11) of the four-vector potential $A_{a v}$ we obtain the gauge transformation behavior

$$
\delta A_{a v}=\frac{\partial \Lambda_{a}}{\partial x^{v}}+\tilde{g} f_{a}^{b c} A_{b v} \Lambda_{c} .
$$


We indeed find the usual gauge transformation behavior for the vector potential of a Yang-Mills theory. The introduction of the parameter $\tilde{g}$ in the definition (11) allows us to explore the full generality of the gauge transformations (23) without changing the normalization of $A_{a v}$.

For a further justification of considering $\tilde{g} \neq 1$, note that the two terms in Eq. (11) have a qualitatively different gauge transformation behavior. In the first term, the derivative acts on the gauge-invariant metric whereas, in the second term, the derivative acts on the gauge-dependent tetrad. Moreover, whenever we can impose the symmetry condition $b_{\kappa \lambda}=b_{\lambda \kappa}$ for $b_{\kappa \mu}=\eta_{\kappa \lambda} b_{\mu}^{\lambda}$, only the first term in Eq. (11) contributes to the linearization of the metric $g_{\mu \nu}$ around the Minkowski metric $\eta_{\mu \nu}$, whereas the second term affects only the nonlinear behavior. Therefore, the value of $\tilde{g}$ has no influence on the weak-field approximation or on the limit of Newtonian gravity. To control these significantly different terms separately, we have introduced the factor $1 / \tilde{g}$ in Eq. (11). Note that the factor $\tilde{g}$ between the two different contributions in Eq. (11) can not be absorbed in the overall normalization of $A_{a v}$.

The gauge transformation (23) of the vector potential implies the standard transformation behavior of the field tensor (8),

$$
\delta F_{a \mu \nu}=\tilde{g} f_{a}^{b c} F_{b \mu \nu} \Lambda_{c},
$$

where the identity (7) has been used. The tensors defined in Eq. (21) possess the gauge transformation behavior

$$
\delta \Delta^{a \mu \nu}=-\tilde{g} f_{b}^{a c} \Delta^{b \mu \nu} \Lambda_{c} .
$$

In view of Eqs. (24) and (25), the Ricci tensor (20) is gauge invariant. Note that this result holds for all values of $\tilde{g}$, not just for $\tilde{g}=1$.

In closing this section on gauge transformations, we mention a particularly convenient gauge for the subsequent developments. If one has $g_{0 j}=0$, then one can choose also $b^{0}{ }_{j}=b^{j}{ }_{0}=0$ (by boosting), $b^{0}{ }_{0}=\sqrt{-g_{00}}$, and the spatial block of $b^{\kappa}{ }_{\mu}$ as the unique positive-definite square root of the spatial block of $g_{\mu \nu}$ (by polar decomposition and rotation). We refer to this convenient possibility as the symmetric gauge [in view of the nonsymmetric role of the two indices of $b^{\kappa}{ }_{\mu}$ in Eq. (12), this possibility is quite remarkable].

\section{GENERAL STATIC ISOTROPIC SOLUTION}

By using the composition rule (11) together with the Yang-Mills theory based on the Lorentz group, we obtain an interesting example of a composite field theory. As the key ingredient is the tetrad decomposition of the metric, which comes with the Lorentz group of gauge transformations, it is natural to evaluate this composite field theory as a possible candidate for an alternative theory of gravity. We do this by studying the counterpart of the general static isotropic metric of general relativity.

In view of the general insights into composite theories reviewed in Sec. II (more precisely, the postprocessing strategy implied by the natural constraints), we first characterize the general structure of isotropic solutions on the level of the variables $A_{a v}$. We then provide the coupled second-order ordinary differential equations for the two functions characterizing the static isotropic solutions of the Yang-Mills theory.
Solutions to these equations can be obtained by series expansion, in the weak-coupling limit, or by numerical integration. We actually find an interesting exact solution, which is the counterpart of the Schwarzschild solution in general relativity. We finally discuss the static isotropic metric $g_{\mu \nu}$ obtained by postprocessing of the solution of the workhorse Yang-Mills theory.

\section{A. Form of static isotropic solution}

The classical high-precision tests of Einstein's theory of gravity are based on the general form of the static isotropic metric produced by a point mass at the origin. The "standard form" of the static isotropic metric in "quasi-Minkowskian coordinates" is given by (see Sec. 8.1 of [18] for details)

$$
g_{\mu \nu}=\left(\begin{array}{cc}
-B & 0 \\
0 & \delta_{m n}+(A-1) x_{m} x_{n} / r^{2}
\end{array}\right),
$$

where $A$ and $B$ are functions of $r=\left(x_{1}^{2}+x_{2}^{2}+x_{3}^{2}\right)^{1 / 2}$. For the decomposition (12), the symmetric gauge leads to

$$
b_{\mu}^{\kappa}=\left(\begin{array}{cc}
\sqrt{B} & 0 \\
0 & \delta_{k m}+(\sqrt{A}-1) x_{k} x_{m} / r^{2}
\end{array}\right),
$$

with the inverse

$$
\bar{b}_{\kappa}^{\mu}=\left(\begin{array}{cc}
1 / \sqrt{B} & 0 \\
0 & \delta_{m k}-(1-1 / \sqrt{A}) x_{m} x_{k} / r^{2}
\end{array}\right) .
$$

The corresponding gauge vector fields (11) are of the following form:

$$
\begin{aligned}
& A_{a 0}=Z\left(x_{1} \quad x_{2} \quad x_{3} \quad 0 \quad 000\right), \\
& A_{a 1}=Y\left(\begin{array}{llllll}
0 & 0 & 0 & 0 & -x_{3} & x_{2}
\end{array}\right), \\
& A_{a 2}=Y\left(\begin{array}{llllll}
0 & 0 & 0 & x_{3} & 0 & -x_{1}
\end{array}\right), \\
& A_{a 3}=Y\left(\begin{array}{llllll}
0 & 0 & 0 & -x_{2} & x_{1} & 0
\end{array}\right) \text {, }
\end{aligned}
$$

with

$$
Y=\frac{1}{\tilde{g} r^{2}}\left[1-(1-\tilde{g}) \frac{\sqrt{A}}{2}-\frac{1+\tilde{g}}{2 \sqrt{A}}\right], \quad Z=\frac{1}{2} \frac{1}{\sqrt{A B}} \frac{B^{\prime}}{r},
$$

where a prime on a function of $r$ indicates the derivative with respect to $r$. Equation (29) represents the static isotropic form of the gauge vector fields satisfying the Lorentz gauge in terms of the two functions $Y(r)$ and $Z(r)$. These functions must be obtained from the Yang-Mills theory. Once the workhorse theory has been solved, Eq. (30) provides the link to a static isotropic metric that can be compared to the corresponding result from general relativity.

\section{B. Field equations}

As the field tensor associated with the vector field in Eq. (29) is antisymmetric in $\mu$ and $v$, it is sufficient to specify it for six index pairs $b$, which we choose according to Table I. The $6 \times 6$ matrix $F_{a b}$ obtained from Eq. (29) according to the definition (8) is of a block-diagonal form, where the two $3 \times 3$ blocks associated with the boosts and rotations are of the form $G_{\mathrm{L}, \mathrm{R}} \delta_{j k}+H_{\mathrm{L}, \mathrm{R}} x_{j} x_{k}$, and the subscripts "L" and "R" stand for 
Lorentz and rotation (or left and right). These functions of $r$ are given by

$$
\begin{aligned}
& G_{\mathrm{L}}=-Z+\tilde{g} r^{2} Y Z, \quad H_{\mathrm{L}}=-\frac{Z^{\prime}}{r}-\tilde{g} Y Z, \\
& G_{\mathrm{R}}=-r Y^{\prime}-2 Y, \quad H_{\mathrm{R}}=\frac{Y^{\prime}}{r}+\tilde{g} Y^{2} .
\end{aligned}
$$

The field equations (10) lead to the following differential equations:

$$
\begin{gathered}
\frac{G_{\mathrm{L}}^{\prime}}{r}+r H_{\mathrm{L}}^{\prime}+4 H_{\mathrm{L}}+2 \tilde{g} Y G_{\mathrm{L}}=0, \\
\frac{G_{\mathrm{R}}^{\prime}}{r}-H_{\mathrm{R}}+\tilde{g}\left(r^{2} Y H_{\mathrm{R}}+Y G_{\mathrm{R}}+Z G_{\mathrm{L}}\right)=0 .
\end{gathered}
$$

When the expressions (31) and (32) are inserted into these first-order differential equations, we obtain the following second-order differential equations for the functions $Y$ and $Z$ characterizing the vector potentials:

$$
\begin{gathered}
Y^{\prime \prime}+4 \frac{Y^{\prime}}{r}+\tilde{g}\left(3-\tilde{g} r^{2} Y\right) Y^{2}+\tilde{g}\left(1-\tilde{g} r^{2} Y\right) Z^{2}=0, \\
Z^{\prime \prime}+4 \frac{Z^{\prime}}{r}+2 \tilde{g}\left(2-\tilde{g} r^{2} Y\right) Y Z=0 .
\end{gathered}
$$

Equations (35) and (36) constitute the Yang-Mills equations for a trial solution of the form (29), which we have recognized as the general static isotropic solution. For reasons of completeness, the explicit expressions for the static isotropic form of the tensors $\Delta^{a \mu v}$ and $R^{\mu}{ }_{v}$ are given in Appendix A.

\section{An exact solution}

If we make the appealing choice $Y=Z$ in Eq. (29), the two coupled ordinary differential equations (35) and (36) reduce to one single differential equation

$$
Y^{\prime \prime}+4 \frac{Y^{\prime}}{r}+2 \tilde{g}\left(2-\tilde{g} r^{2} Y\right) Y^{2}=0 .
$$

For the choice $Y=Z$, Eq. (30) implies that $A$ and $B$ are related by

$$
\sqrt{A}-1-\frac{1}{2}(1-\tilde{g})(A-1)=\tilde{g} r \frac{d \sqrt{B}}{d r} .
$$

Quite remarkably, the nonlinear equation (37) possesses the closed-form solutions

$$
Y=\frac{1}{r^{2}\left(\tilde{g}+r / r_{0}\right)},
$$

where $r_{0}$ is an arbitrary constant. From Eq. (30) [or, more conveniently, by inserting $Y$ into Eq. (A6)], we obtain the exact result for $A$ :

$$
\sqrt{A}=\frac{1-\operatorname{sign}(\tilde{g}) \sqrt{1-\left(1-\tilde{g}^{2}\right)\left(1+\tilde{g} r_{0} / r\right)^{2}}}{(1-\tilde{g})\left(1+\tilde{g} r_{0} / r\right)} .
$$

Finally, the exact result for $B$ can be obtained by integrating the expression for $Z$ in Eq. (30) [or from Eq. (A7)]:

$$
\begin{aligned}
\sqrt{B}-1= & \frac{\sqrt{A}-1}{\tilde{g}}+\frac{1}{|\tilde{g}|} \sqrt{\frac{1+\tilde{g}}{1-\tilde{g}}} \\
& \times\left\{\arccos \left(\sqrt{1-\tilde{g}^{2}}\left[1+\tilde{g} \frac{r_{0}}{r}\right]\right)-\arcsin (|\tilde{g}|)\right\} .
\end{aligned}
$$

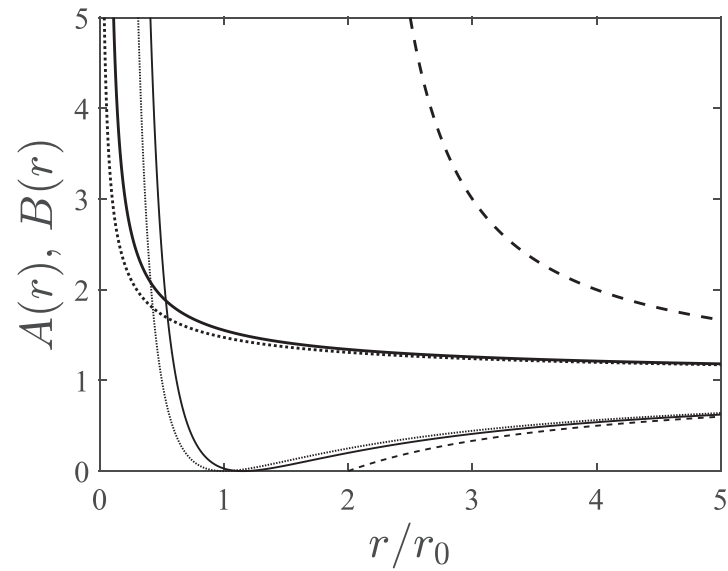

FIG. 1. The functions $A$ (thick lines) and $B$ (thin lines) characterizing the isotropic metric (26) obtained from the composite theory for $\tilde{g}=-0.03$. The continuous lines represent the exact results (40) and (41), whereas the dotted lines represent the weak-coupling limits (49) and (50). The dashed lines represent the Schwarzschild solution of general relativity for comparison.

For any value of the coupling constant $\tilde{g}$, Eqs. (40) and (41) provide a one-parameter family of exact solutions for the static isotropic metric (26) predicted by our composite higher derivative theory of gravity. This remarkable result is the counterpart of the Schwarzschild solution in general relativity, which is given by

$$
A=\left(1-2 \frac{M G}{r}\right)^{-1}, \quad B=1-2 \frac{M G}{r},
$$

where $M$ is the mass at the origin producing the isotropic field and $G$ is Newton's constant. The product $2 M G$ is known as the Schwarzschild radius, which is closely related to the parameter $r_{0}$ in the solution (40) and (41) [see Eq. (47) below].

For $0<\tilde{g}<1$, the solution (40) becomes imaginary for $r<r_{+}$with

$$
r_{+}=\left(1-\tilde{g}^{2}+\sqrt{1-\tilde{g}^{2}}\right) \frac{r_{0}}{\tilde{g}},
$$

that is, when $A$ reaches the finite value $(1+\tilde{g}) /(1-\tilde{g})$ and the slope becomes infinite. For $\tilde{g}<0, Y$ and $A$ diverge at

$$
r_{-}=|\tilde{g}| r_{0} \text {. }
$$

The results for $A$ and $B$ for the coupling constant $\tilde{g}=-0.03$ are shown in Fig. 1, together with the Schwarzschild solution (for which $A$ diverges at the Schwarzschild radius $2 r_{0}$ rather than the much smaller value $\left.|\tilde{g}| r_{0}\right)$.

\section{Robertson expansions}

The three classical tests of general relativity suggested by Einstein are (i) the gravitational redshift of spectral lines from white dwarf stars, (ii) the deflection of light (or electromagnetic waves outside the visible spectrum) by the sun, and (iii) the anomalous precession of the perihelion of mercury. A further testable prediction is (iv) the travel time delay for radar signals reflecting off other planets. All these high-precision tests for theories of gravity probe the asymptotic behavior of $A$ and $B$ for large $r$. Experimental results can conveniently be 
analyzed on the basis of the standard Robertson expansions (see, e.g., Sec. 8.3 of [18]),

$$
\begin{aligned}
& A=1+2 \gamma \frac{M G}{r}+\cdots, \\
& B=1-2 \frac{M G}{r}+2(\beta-\gamma) \frac{M^{2} G^{2}}{r^{2}}+\cdots .
\end{aligned}
$$

From these expansions of $A$ and $B$, we obtain the corresponding leading-order terms for the functions $Y$ and $Z$ defined in Eq. (30):

$$
\begin{aligned}
& Y=\gamma \frac{M G}{r^{3}}+\cdots \\
& Z=\frac{M G}{r^{3}}-(2 \beta-\gamma-1) \frac{M^{2} G^{2}}{r^{4}}+\cdots .
\end{aligned}
$$

Assuming $Y=Z$, the exact solution (39) implies

$$
r_{0}=M G, \quad \beta=1+\frac{\tilde{g}}{2}, \quad \gamma=1 .
$$

We recognize $r_{0}$ as half of the Schwarzschild radius. The extremely successful predictions of Einstein's theory of general relativity are $\beta=\gamma=1$, so that the coupling constant $\tilde{g}$ must be close to zero (modern results require a very small coupling, $|\tilde{g}| \lesssim 2 \times 10^{-4}$ ). In view of the remarks at the end of Sec. V C, it is preferable to let $\tilde{g}$ go to zero from below. If we allow $Y$ to be different from $Z$, Eq. (36) leads to the identity

$$
2 \beta-(1+\tilde{g}) \gamma-1=0,
$$

which is, of course, satisfied by the values given in (47).

\section{E. Limit of small $\tilde{g}$}

We have seen that consistency with experimental observations requires small values of $\tilde{g}$. In the limit of small $\tilde{g}$, Eq. (38) becomes $r_{0} / r=r d \sqrt{B} / d r$ so that we find

$$
\sqrt{B}=1-\frac{r_{0}}{r} \text {. }
$$

The exact partner $\sqrt{A}$ for this limiting result for $\sqrt{B}$ according to Eq. (38) is

$$
\sqrt{A}=\frac{1}{1-\tilde{g}}\left[1-\tilde{g} \sqrt{1-2 \frac{1-\tilde{g}}{\tilde{g}} \frac{r_{0}}{r}}\right] .
$$

Of course, in the limit of small $\tilde{g}$, the factors $1-\tilde{g}$ in Eq. (50) can be replaced by 1 . These limiting results are compared to the exact results for $A$ and $B$ in Fig. 1. Contrary to the Schwarzschild solution of general relativity, for which $B$ is negative for $r<2 r_{0}, B$ never becomes negative for the composite theory (but $B$ becomes 0 at the single point $r=r_{0}$, where the proper time does not change with background time).

\section{SUMMARY AND CONCLUSIONS}

We have considered a composite higher derivative theory in which the variables of the Yang-Mills theory based on the Lorentz group are expressed in terms of the tetrad decomposition of a metric. The composition rule (11) introduces an adjustable coupling constant into the Yang-Mills theory, which is the key innovation of the present approach. The composite theory is proposed and tested as an alternative theory of gravity. For small coupling constants, the highprecision predictions of Einstein's general theory of relativity are fully reproduced.

The most important result for our alternative theory of gravity is the exact static isotropic solution for all values of the coupling constant $\tilde{g}$, which is the counterpart of the Schwarzschild solution in general relativity. If we approach $\tilde{g}=0$ from below, this exact solution diverges only at the origin. The only irregularity existing for $r>0$ is that the element $g_{00}$ of the metric becomes zero if $r$ is equal to one half of the Schwarzschild radius, so that the proper time stands still at this distance from the origin.

Another important feature of general relativity is the prediction of gravitational waves, which have eventually been detected in 2015. In the context of our composite higher derivative theory of gravity, such waves have already been discussed in Appendix B of [1]. Again, the results are found to be fully consistent with general relativity.

For the present discussion of static isotropic solutions, and also for the previous discussion of gravitational waves, we have considered only the composite higher derivative theory for pure gravity. Of course, the coupling to matter in this new type of theory is of crucial importance. The following two gauge-invariant extensions of the dimensionless action (9) are promising candidates for the scalar or tensorial coupling of the gravitational field to matter (note that the "general wisdom" about the possibilities of coupling gravity to matter is not beyond all doubt [26-29]),

$$
I=\int\left\{-\frac{1}{4} F_{a \mu \nu} F^{a \mu \nu}+\frac{2 \pi G}{c^{4}}\left(\ell^{-2}+R\right) T_{\mu}{ }^{\mu}\right\} d^{4} x
$$

or

$I=\int\left\{-\frac{1}{4} F_{a \mu \nu} F^{a \mu \nu}+\frac{2 \pi G}{c^{4}}\left[\left(\ell^{-2}+R\right) \delta^{\mu}{ }_{\nu}-4 R_{\nu}^{\mu}\right] T_{\mu}{ }^{\nu}\right\} d^{4} x$,

where the curvature tensor $R^{\mu}{ }_{\nu}$ has been defined in Eq. (20), $R=R_{\mu}^{\mu}$ is its trace, and $\ell$ is a length scale that regularizes the action for vanishing curvature. Matter is characterized by the energy-momentum tensor $T_{\mu}{ }^{\nu}$. In this energy-momentum tensor, momenta should be constructed from velocities by means of the metric $g_{\mu \nu}$. Also, the kinetic energy, which is quadratic in the velocity, should be formed with $g_{\mu \nu}$. In the present approach, it is more appropriate to consider $g_{\mu \nu}$ as a tool for converting scalar mass into a tensorial property, not as a metric in space. The geometric significance of $g_{\mu \nu}$ is to relate tangent vector spaces to their dual vector spaces. In Appendix B, we sketch how one can derive the weak-field equations arising from the actions (51) and (52), respectively. The action (51) with scalar coupling contains the proper limit of Newtonian gravity, whereas Eq. (52) for tensorial coupling is even closer to general relativity. Of course, these actions also characterize the motion of matter in a gravitational field. According to the results of Appendix A [cf. Eq. (A8)], geodesic motion for a mass point in a static isotropic gravitational field is obtained only for $\ell r_{0} \ll r^{2}$ if we use the tensorial coupling in the action (52) whereas, for the action (51) with scalar coupling, $\ell$ can be arbitrarily large because $R$ is zero. For example, $\ell$ could then be taken as the size of the universe, which would actually 
make this alternative theory of gravity weakly time dependent and might imply some interesting features of gravity on very large scales. The energy-momentum tensor expressing the local energy-momentum conservation associated with the action (51) is discussed in Appendix C.

For composite higher derivative theories, there exist a natural canonical Hamiltonian formulation and a natural set of constraints [2]. In other words, composite Yang-Mills theories provide a promising setting for quantization. Contrary to the effective field theories for the electroweak and strong interactions with an arbitrary cutoff that requires renormalization, the proposed composite higher derivative theory for gravity should be regularized by a physical mechanism on the Planck scale. As suggested in [30], this can be achieved very robustly in the setting of dissipative quantum field theory, where the regularization is provided by dissipative smearing.

Of course, the elegant beauty of Einstein's general theory of relativity is unbeatable. However, the Yang-Mills theory based on the Lorentz group is an attractive theory for gravity rooted in geometry. Using that Yang-Mills theory as the workhorse for a composite theory with a natural composition rule mapping tetrad variables into gauge vector fields provides an appealing overall scenario for a theory of gravity.

\section{APPENDIX A: CURVATURE OF STATIC ISOTROPIC SOLUTION}

As the tensor $\Delta^{a \mu \nu}$ defined in Eq. (21) is antisymmetric in $\mu$ and $\nu$, it is sufficient to specify it for six index pairs $b$, which we choose according to Table I. Exactly as we had noted for the field tensor in Sec. V B, the $6 \times 6$ matrix $\Delta^{a b}$ is of a blockdiagonal form, where the two $3 \times 3$ blocks associated with the Lorentz boosts and rotations for $\Delta^{a b}$ are given by

$$
\Delta_{\mathrm{L}}^{m n}=\frac{1}{\sqrt{B}}\left[\delta_{m n}-\left(1-\frac{1}{\sqrt{A}}\right) \frac{x_{m} x_{n}}{r^{2}}\right]
$$

and

$$
\Delta_{\mathrm{R}}^{m n}=-\frac{1}{\sqrt{A}} \delta_{m n}-\left(1-\frac{1}{\sqrt{A}}\right) \frac{x_{m} x_{n}}{r^{2}} .
$$

The Ricci curvature tensor now follows from Eq. (20). As the components for mixed space and time indices vanish $\left(R_{n}^{0}=R^{m}{ }_{0}=0\right)$, we only need to list the nonvanishing components

$$
R_{0}^{0}=-\frac{1}{\sqrt{B}}\left[G_{\mathrm{L}}\left(2+\frac{1}{\sqrt{A}}\right)+H_{\mathrm{L}} \frac{r^{2}}{\sqrt{A}}\right]
$$

and

$$
\begin{aligned}
R_{n}^{m}= & -\frac{1}{\sqrt{B}} G_{\mathrm{L}}\left[\delta_{m n}-\left(1-\frac{1}{\sqrt{A}}\right) \frac{x_{m} x_{n}}{r^{2}}\right] \\
& +G_{\mathrm{R}}\left(1+\frac{1}{\sqrt{A}}\right) \delta_{m n}-G_{\mathrm{R}}\left(1-\frac{1}{\sqrt{A}}\right) \frac{x_{m} x_{n}}{r^{2}} \\
& -H_{\mathrm{L}} \frac{x_{m} x_{n}}{\sqrt{A B}}+H_{\mathrm{R}}\left(r^{2} \delta_{m n}-x_{m} x_{n}\right) .
\end{aligned}
$$

For the curvature scalar, we then find

$$
\begin{aligned}
R=R_{\mu}^{\mu}=2[ & \left.G_{\mathrm{R}}\left(1+\frac{2}{\sqrt{A}}\right)+H_{\mathrm{R}} r^{2}\right] \\
& -\frac{2}{\sqrt{B}}\left[G_{\mathrm{L}}\left(2+\frac{1}{\sqrt{A}}\right)+H_{\mathrm{L}} \frac{r^{2}}{\sqrt{A}}\right] .
\end{aligned}
$$

Note that, once the functions $Y$ and $Z$ have been determined, the calculation of the functions $G_{\mathrm{L}, \mathrm{R}}$ and $H_{\mathrm{L}, \mathrm{R}}$ from Eqs. (31) and (32) is straightforward. From Eq. (30), we moreover obtain

$$
\sqrt{A}=\frac{1-\tilde{g} r^{2} Y-\operatorname{sign}(\tilde{g}) \sqrt{\left(1-\tilde{g} r^{2} Y\right)^{2}-1+\tilde{g}^{2}}}{1-\tilde{g}},
$$

where the sign of the square root is chosen such that $Y \rightarrow 0$ implies $A \rightarrow 1$ (Minkowski background). Finally, we obtain $B$ by integration,

$$
\sqrt{B}=\int r Z \sqrt{A} d r
$$

where the integration constant is to be chosen such that $B \rightarrow 1$ for $r \rightarrow \infty$.

In the limit of vanishing coupling constant $\tilde{g}$, only the spatial components of the curvature tensor are nonvanishing,

$$
R_{n}^{m}=\frac{r_{0}^{2}}{r^{3}\left(r-r_{0}\right)}\left(\delta_{m n}-3 \frac{x_{m} x_{n}}{r^{2}}\right) .
$$

In addition to $R_{0}^{0}=0$, we find that also the curvature scalar vanishes, $R=0$.

\section{APPENDIX B: WEAK-FIELD APPROXIMATION}

We here develop the weak-field approximation which, in particular, allows one to consider gravitational waves (which have previously been discussed for the composite higher derivative theory in Appendix B of [1]) and the limit of Newtonian gravity. We assume the metric to be close to the background Minkowski metric

$$
g_{\mu \nu}=\eta_{\mu \nu}+h_{\mu \nu} .
$$

In the symmetric gauge discussed at the end of Sec. IV C, we obtain to first order in $h$

$$
b_{\mu}^{\kappa}=\delta_{\mu}^{\kappa}+\frac{1}{2} h_{\mu}^{\kappa},
$$

and the definition (11) leads to the four-vector potential

$$
A_{a v}=\frac{1}{2}\left(\frac{\partial h_{\tilde{\lambda} v}}{\partial x^{\tilde{\kappa}}}-\frac{\partial h_{\tilde{\kappa} v}}{\partial x^{\tilde{\lambda}}}\right) .
$$

As anticipated in Sec. IV B, the vector potential of the weakfield approximation does not depend on $\tilde{g}$. Also, the field tensor defined in Eq. (8) is independent of $\tilde{g}$ :

$$
F_{a \mu \nu}=\frac{1}{2}\left(\frac{\partial^{2} h_{\tilde{\lambda} \nu}}{\partial x^{\mu} \partial x^{\tilde{\kappa}}}-\frac{\partial^{2} h_{\tilde{\kappa} \nu}}{\partial x^{\mu} \partial x^{\tilde{\lambda}}}-\frac{\partial^{2} h_{\tilde{\lambda} \mu}}{\partial x^{\nu} \partial x^{\tilde{\kappa}}}+\frac{\partial^{2} h_{\tilde{\kappa} \mu}}{\partial x^{\nu} \partial x^{\tilde{\lambda}}}\right) .
$$


From Eq. (20) we obtain the Ricci curvature tensor

$R_{\nu}^{\mu}=F_{\nu \lambda}^{\mu \lambda}=\frac{1}{2}\left(\frac{\partial^{2} h_{\lambda}^{\lambda}}{\partial x_{\mu} \partial x^{\nu}}-\frac{\partial^{2} h_{\lambda}^{\mu}}{\partial x_{\lambda} \partial x^{\nu}}-\frac{\partial^{2} h_{\nu}^{\lambda}}{\partial x_{\mu} \partial x^{\lambda}}+\frac{\partial^{2} h^{\mu}{ }_{\nu}}{\partial x_{\lambda} \partial x^{\lambda}}\right)$,

which coincides with Eq. (7.6.2) of [18]. A further contraction gives the curvature scalar

$$
R=\frac{\partial^{2} h_{\mu}^{\mu}}{\partial x_{v} \partial x^{\nu}}-\frac{\partial^{2} h_{\nu}^{\mu}}{\partial x_{v} \partial x^{\mu}}
$$

These lowest-order expressions in $h_{\mu \nu}$ imply the following functional derivatives:

$$
\begin{aligned}
& \frac{\delta}{\delta h^{\mu \nu}} \int R X d^{4} x=\left(\eta_{\mu \nu} \square-\frac{\partial^{2}}{\partial x^{\mu} \partial x^{\nu}}\right) X, \\
& \frac{\delta}{\delta h^{\mu \nu}} \int R_{v^{\prime}}^{\mu^{\prime}} X_{\mu^{\prime}} v^{v^{\prime}} d^{4} x \\
& =\frac{1}{2}\left(\square X_{\mu \nu}+\eta_{\mu \nu} \frac{\partial^{2} X_{\mu^{\prime}}{ }^{\nu^{\prime}}}{\partial x_{\mu^{\prime}} \partial x^{\nu^{\prime}}}-\frac{\partial^{2} X_{\mu}{ }^{\lambda}}{\partial x^{\nu} \partial x^{\lambda}}-\frac{\partial^{2} X_{\nu}^{\lambda}}{\partial x^{\mu} \partial x^{\lambda}}\right),
\end{aligned}
$$

$$
\frac{\delta}{\delta h^{\mu \nu}} \frac{1}{2} \int F_{a \mu^{\prime} v^{\prime}} F^{a \mu^{\prime} v^{\prime}} d^{4} x=\frac{\partial^{2} F_{\kappa \mu, \lambda \nu}}{\partial x_{\kappa} \partial x_{\lambda}}=\square R_{\mu \nu}-\frac{1}{2} \frac{\partial^{2} R}{\partial x^{\mu} \partial x^{\nu}},
$$

where $\square=\partial^{2} / \partial x^{\mu} \partial x_{\mu}$. According to these results for the weak-field approximation, the action (52) with tensorial coupling, for negligible regularization effects, implies the following field equations:

$$
\begin{aligned}
& \square\left[R_{\mu \nu}+\frac{8 \pi G}{c^{4}}\left(T_{\mu \nu}-\frac{1}{2} \eta_{\mu \nu} T_{\lambda}^{\lambda}\right)\right] \\
& \quad-\frac{1}{2} \frac{\partial^{2}}{\partial x^{\mu} \partial x^{\nu}}\left(R_{\lambda}^{\lambda}-\frac{8 \pi G}{c^{4}} T_{\lambda}^{\lambda}\right)=0 .
\end{aligned}
$$

This result is consistent with Einstein's general theory of relativity. The solutions of the higher derivative equations (B10) still need to be restricted by constraints.

From the action (51) with scalar coupling, we obtain the following field equations:

$$
\square R_{\mu \nu}-\frac{1}{2} \frac{\partial^{2} R}{\partial x^{\mu} \partial x^{\nu}}-\frac{4 \pi G}{c^{4}}\left(\eta_{\mu \nu} \square-\frac{\partial^{2}}{\partial x^{\mu} \partial x^{\nu}}\right) T_{\lambda}^{\lambda}=0 .
$$

For time-independent mass densities $\rho$, we expect $T_{\lambda}{ }^{\lambda}=\rho c^{2}$. If we then define a dimensionless Newtonian potential $\phi$ through the Poisson equation $\square \phi=4 \pi G \rho / c^{2}$, Eq. (B11) can easily be solved in two steps:

$$
R_{\mu \nu}=\eta_{\mu \nu} \square \phi+2 \frac{\partial^{2} \phi}{\partial x^{\mu} \partial x^{\nu}}, \quad h_{\mu \nu}=2 \eta_{\mu \nu} \phi .
$$

The result $h_{00}=-2 \phi$ implies $\Gamma_{00}^{\mu}=\partial \phi / \partial x_{\mu}$ so that geodetic motion at small velocities reproduces Newton's theory of gravity.

The role of constraints in solving fourth-order differential equations in the context of the weak-field approximation for pure gravity and, in particular, of gravitational waves has been discussed in Appendix B of [1]. The respective form of the natural primary constraints suggested for the Ostrogradsky approach have been given in Eqs. (B11) and (B13) of [1]. For gravitational waves, it has been shown explicitly how the secondary constraints can be used to eliminate fourth derivatives with respect to time to obtain second-order differential equations in time.

\section{APPENDIX C: ENERGY-MOMENTUM CONSERVATION}

Let us consider the Lagrangian density

$$
\mathcal{L}=-\frac{c^{4} \ell^{2}}{8 \pi G} F_{a \mu \nu} F^{a \mu \nu}+\left(1+\ell^{2} R\right) T_{\mu}^{\mu}
$$

Note that the integral $\hat{I}=\int \mathcal{L} d^{4} x$ differs from the dimensionless action $I$ defined in Eq. (51) only by a constant factor. According to Eqs. (8), (11) and (20), (21), $F$ and $R$ depend on $b^{\kappa}{ }_{\mu}$ and its first and second derivatives whereas, according to the remarks after Eq. (51), $T_{\mu}^{\mu}=T_{\mu}^{\mu}\left(g_{\mu^{\prime} v^{\prime}}(x), x\right)$ depends only on $b^{\kappa}{ }_{\mu}$ itself, and in addition also explicitly on $x$. Of course, this explicit dependence on $x$ is not an external one but is rather associated with the distribution of matter in the system (e.g., along the world line of a particle).

In order to construct an energy-momentum balance, we calculate

$$
\frac{\partial \mathcal{L}}{\partial x^{\mu}}-\frac{\delta \hat{I}}{\delta b^{\kappa}{ }_{\nu}} \frac{\partial b^{\kappa}{ }_{\nu}}{\partial x^{\mu}}=-\frac{\partial \hat{T}_{\mu}^{\nu}}{\partial x^{\nu}}+\left(1+\ell^{2} R\right) \frac{\partial T_{\nu}{ }^{\nu}}{\partial x^{\mu}},
$$

with

$$
-\hat{T}_{\mu}^{\nu}=b_{\mu^{\prime}, \mu}^{\kappa} \frac{\partial \mathcal{L}}{\partial b^{\kappa}{ }_{\mu^{\prime}, \nu}}+\left(b_{\mu^{\prime}, \mu, v^{\prime}}-b^{\kappa}{ }_{\mu^{\prime}, \mu} \frac{\partial}{\partial x^{v^{\prime}}}\right) \frac{\partial \mathcal{L}}{\partial b^{\kappa}{ }_{\mu^{\prime}, \nu, v^{\prime}}},
$$

where we have used the convenient comma notation for the partial derivatives of $b^{\kappa}{ }_{\mu^{\prime}}$ to keep the expression for $\hat{T}_{\mu}^{\nu}$ readable. Note that only the derivatives of $b^{\kappa}{ }_{\mu^{\prime}}$ contribute to the energy-momentum tensor, that is, the quadratic occurrence of the field tensor $F$ in the first term of Eq. (C1) (pure gravity) and the implicit linear occurrence of $F$ in $R$ (coupling to matter). We here refrain from producing a symmetric energy-momentum tensor $\hat{T}_{\mu}^{v}$ by the standard Belinfante procedure [31]. The last term in Eq. (C2) results from the explicit dependence of $T_{\nu}{ }^{v}$ on $x$ for fixed metric.

For solutions of the field equations, $\delta \hat{I} / \delta b^{\kappa}{ }_{v}$ vanishes and Eq. (C2) is simplified. If we further assume $R=0$ (as we found at the end of Appendix A for the static isotropic solution), Eq. (C2) takes the form of the local energy-momentum conservation expected according to Noether's theorem from the invariance of the action under space-time translations. A divergence-free energy-momentum tensor $T_{\mu}{ }^{\nu}$ of matter is not required for or implied by this analysis. A vanishing divergence of $T_{\mu}{ }^{v}$ rather needs to be established by separate arguments (see, e.g., Secs. 5.3 and 5.4 of [18]). For other couplings between gravitational field and matter, restrictions might arise. 
[1] H. C. Öttinger, Hamiltonian formulation of a class of constrained fourth-order differential equations in the Ostrogradsky framework, J. Phys. Commun. 2, 125006 (2018).

[2] H. C. Öttinger, Natural Hamiltonian formulation of composite higher derivative theories, J. Phys. Commun. 3, 085001 (2019).

[3] S. Capozziello and M. De Laurentis, Extended theories of gravity, Phys. Rep. 509, 167 (2011).

[4] M. Ostrogradsky, Mémoires sur les équations différentielles, relatives au problème des isopérimètres, Mem. Acad. St. Petersb. 6, 385 (1850).

[5] R. P. Woodard, The theorem of Ostrogradsky, arXiv:1506.02210.

[6] T. J. Chen, M. Fasiello, E. A. Lim, and A. J. Tolley, Higher derivative theories with constraints: Exorcising Ostrogradski's ghost, J. Cosmol. Astropart. Phys. 02 (2013) 042.

[7] M. Raidal and H. Veermäe, On the quantisation of complex higher derivative theories and avoiding the Ostrogradsky ghost, Nucl. Phys. B 916, 607 (2017).

[8] K. S. Stelle, Renormalization of higher-derivative quantum gravity, Phys. Rev. D 16, 953 (1977).

[9] K. S. Stelle, Classical gravity with higher derivatives, Gen. Relat. Gravit. 9, 353 (1978).

[10] N. V. Krasnikov, Nonlocal gauge theories, Theor. Math. Phys. 73, 1184 (1987).

[11] C. Grosse-Knetter, Effective Lagrangians with higher derivatives and equations of motion, Phys. Rev. D 49, 6709 (1994).

[12] D. Becker, C. Ripken, and F. Saueressig, On avoiding Ostrogradski instabilities within asymptotic safety, J. High Energy Phys. 12 (2017) 121.

[13] A. Salvio, Metastability in quadratic gravity, Phys. Rev. D 99, 103507 (2019).

[14] H. Weyl, The Classical Groups: Their Invariants and Representations, 2nd ed. (Princeton University Press, Princeton, 1946).

[15] M. E. Peskin and D. V. Schroeder, An Introduction to Quantum Field Theory (Perseus Books, Reading, MA, 1995).
[16] S. Weinberg, Modern Applications, The Quantum Theory of Fields Vol. 2 (Cambridge University Press, Cambridge, 2005).

[17] H. C. Öttinger, BRST quantization of Yang-Mills theory: A purely Hamiltonian approach on Fock space, Phys. Rev. D 97, 074006 (2018).

[18] S. Weinberg, Gravitation and Cosmology, Principles and Applications of the General Theory of Relativity (Wiley, New York, 1972).

[19] A. Ashtekar, New Variables for Classical and Quantum Gravity, Phys. Rev. Lett. 57, 2244 (1986).

[20] A. Ashtekar, New Hamiltonian formulation of general relativity, Phys. Rev. D 36, 1587 (1987).

[21] C. N. Yang and R. L. Mills, Conservation of isotopic spin and isotopic gauge invariance, Phys. Rev. 96, 191 (1954).

[22] R. Utiyama, Invariant theoretical interpretation of interaction, Phys. Rev. 101, 1597 (1956).

[23] D. Ivanenko and G. Sardanashvily, The gauge treatment of gravity, Phys. Rep. 94, 1 (1983).

[24] C. N. Yang, Integral Formalism for Gauge Fields, Phys. Rev. Lett. 33, 445 (1974).

[25] Gauge Theories of Gravitation: A Reader with Commentaries, edited by M. Blagojević and F. W. Hehl (Imperial College Press, London, 2013).

[26] R. P. Feynman, F. B. Morinigo, and W. G. Wagner, Feynman Lectures On Gravitation (Addison-Wesley, Reading, MA, 1995).

[27] S. Deser, Self-interaction and gauge invariance, Gen. Relat. Gravit. 1, 9 (1970).

[28] N. Straumann, Reflections on gravity, arXiv:astro-ph/0006423.

[29] T. Padmanabhan, From gravitons to gravity: Myths and reality, Int. J. Mod. Phys. D 17, 367 (2008).

[30] H. C. Öttinger, A Philosophical Approach to Quantum Field Theory (Cambridge University Press, Cambridge, 2017).

[31] F. J. Belinfante, On the current and the density of the electric charge, the energy, the linear momentum and the angular momentum of arbitrary fields, Physica (Amsterdam) 7, 449 (1940). 